Alper, Tikvah (1954). J. gen. Microbiol. 11, 313-324.

\title{
The Inactivation of Free Bacteriophage by Irradiation and by Chemical Agents
}

\author{
BY TIKVAH ALPER \\ Experimental Radiopathology Research Unit, Hammersmith Hospital, \\ Ducane Road, London, W. 12
}

SUMMARY : Shigella phage $S 13$ and coli phage $T 3$ are very sensitive, in the free state, to inactivation by reducing radicals produced in water by ionizing radiation. They are inactivated by ascorbic acid, and by hydrogen peroxide $\left(\mathrm{H}_{2} \mathrm{O}_{2}\right)$ acting as a reductant; but the $\mathrm{H}_{2} \mathrm{O}_{2}$ must decompose into active radicals before it attacks the phage. Hydrogen peroxide decomposes under irradiation, and attacks phage much more vigorously under ionizing radiation than under ultraviolet irradiation. Free radicals may part-inactivate phage, that is, render it more sensitive to $\mathrm{H}_{2} \mathrm{O}_{2}$ and to ascorbic acid, though not to inactivating agents in general. Phage grown on rich medium is more sensitive than phage grown on poor medium to inactivation by reduction.

Some results of general interest in microbiology have emerged from a series of investigations which were undertaken primarily to obtain information on the mode of action of ionizing radiations on bacteriophage. The main interest centred on 'indirect action', that is, the inactivating effects of the decomposition products of irradiated water. Interpretation of the radiation results necessitated a study of the effects of hydrogen peroxide and other inactivating agents on free phage. Certain conclusions have been reached as to the mechanism of inactivation by chemical agents, as well as by irradiation, when the phage is exposed in dilute suspension before adsorption to the bacterial host.

\section{MATERIAL AND METHODS}

Strains of bacteriophage and bacteria. Most of the experiments were done with the shigella phage S13, active against Shigella flexneri strain Y6R; the stock was derived from that used by Lea \& Salaman (1946). For plaque counts a sensitive Escherichia coli strain was used, as it was preferable to work with a non-pathogenic organism. When 'part-inactivation' of phage S13 was discovered (Alper 1952 $a, 1954$ ), experiments were done with the coli phage T3 to see whether or not the phenomenon was general.

Preparation of bacteriophage stocks. For radiation experiments on dilute suspensions, bacteriophage stocks of high titre and low solid content were needed. To this end the phage was propagated on the surface of a solid medium of low nutrient content, made up of $0.5 \%(\mathrm{w} / \mathrm{v})$ Evans peptone and $2 \%(\mathrm{w} / \mathrm{v})$ agar. Petri dishes containing this medium were inoculated first with host cells derived from a single colony, and, after about $2 \mathrm{hr}$. incubation, with a phage suspension of suitable titre. After lysis of the host cells by the phage, the plates were each washed with about $2 \mathrm{ml} .0 .001 \mathrm{M}$-phosphate buffer $(\mathrm{pH} \mathrm{7 \cdot 0})$ 
and the washings centrifuged at 6000 r.p.m. The water-clear supernatant usually had a phage titre of from $5.10^{9}$ to $10^{11}$ particles $/ \mathrm{ml}$, and solid content between 2 and $10 \mathrm{mg} . / \mathrm{ml}$. In most of the experiments, suspensions were used at dilutions of about $10^{-6}$.

The nature of the medium on which the phage was propagated was found to have a bearing on its sensitivity to radiation-produced active radicals and to hydrogen peroxide. For experiments specifically designed to examine this dependence, phage stocks were grown from the same host and phage clones both on peptone agar and on nutrient agar plates. The two types of S13 stock so derived will be referred to as $\mathrm{S} 13 \mathrm{P}$ and $\mathrm{S} 13 \mathrm{R}$ respectively.

Preparation and handling of dilute phage suspensions. To obtain clear-cut and reproducible results on dilute phage suspensions it was necessary to exclude as far as possible traces of organic or inorganic contaminating material. The chemicals used were always of analytical reagent quality; the water used in making the dilute suspensions (about $10^{-6}$ ), the buffer and the other solutions, was twice distilled in glass from alkaline permanganate; glassware was stored in sulphuric + chromic acid cleaning solution, and very thoroughly washed before use, being given three final rinses with glass-distilled water. It was found desirable to eliminate the possibility that volatile substances from rubber teats might be absorbed in the dilute phage suspensions and influence their behaviour. Dropping pipettes were therefore made with ground glass cone terminals and fitted to joints specially made to attach to $1 \mathrm{ml}$. Luer-lock all-glass syringes.

Plaque counting: standard errors. Measured volumes of phage and bacterial suspensions were delivered by calibrated dropping pipettes on to nutrient agar plates and spread together over the surface. The plates were incubated for about $8 \mathrm{hr}$. at $37^{\circ}$, and the plaques then counted. The error of any plaque count included variations in drop size and ordinary statistical fluctuations. By making counts on 25 or more replicates, the standard deviation of any count $n$ was found by experiment, and confirmed by periodic checks, to be $\sqrt{ } 2 n$, instead of the theoretical $\sqrt{ } n$. Thus the standard error of an observation of $m$ replicates, with a mean count of $n$ plaques per replicate, was $\sqrt{ }(2 n / m)$. Standard errors indicated in the diagrams have been calculated on this basis.

Irradiations. Neutrons and electrons from a van de Graaff generator, gamma-rays, and $200 \mathrm{kV}$. X-rays were used to investigate the effects of ionizing radiation. A Hanovia 'Germicidal' lamp, with most of the energy at $2537 \mathrm{~A}$, was used as the source of ultraviolet light. Details will be published elsewhere concerning methods of irradiation and dosimetry, and of bubbling gases through phage suspensions undergoing irradiation.

Estimation of hydrogen peroxide. Interpretation of the radiation data depended on a knowledge of the amount of $\mathrm{H}_{2} \mathrm{O}_{2}$ formed by a given dose of ionizing radiation. Two methods were used: (1) for concentrations above $1 \mu \mathrm{g} . / \mathrm{ml}$., the method of Eisenberg (1943), in which titanous sulphate is oxidized to the titanic form and then to peroxide; (2) for lower concentrations, the method of Savage (1951), in which iodide is oxidized to iodine in the presence of starch, a coloured complex being formed. 


\section{RESULTS}

\section{Effects of hydrogen peroxide}

When coli phage T3 or shigella phage $\mathrm{S} 13$ were exposed to $\mathrm{H}_{2} \mathrm{O}_{2}$, the curves of survival with time were usually of exponential form. The slopes of the curves depended on the conditions of the experiment, such as hydrogen peroxide concentration, temperature, and organic content of the phage suspension (Alper 1952b). Several other variables have now been investigated.

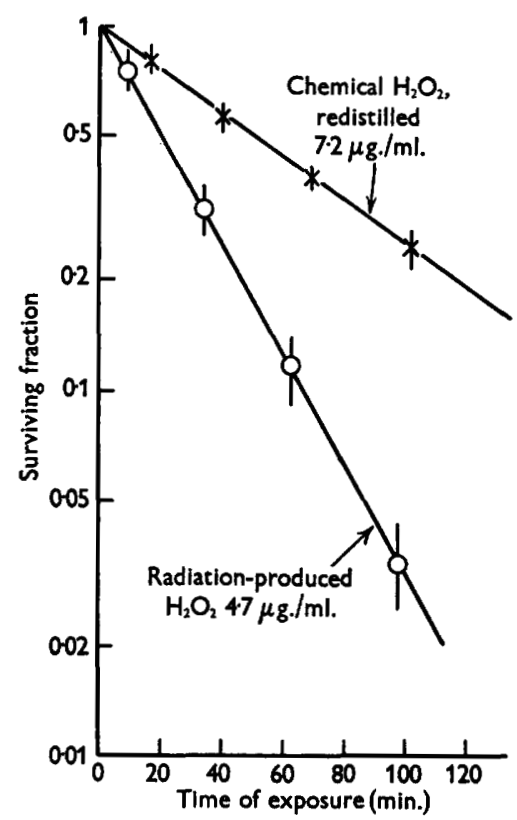

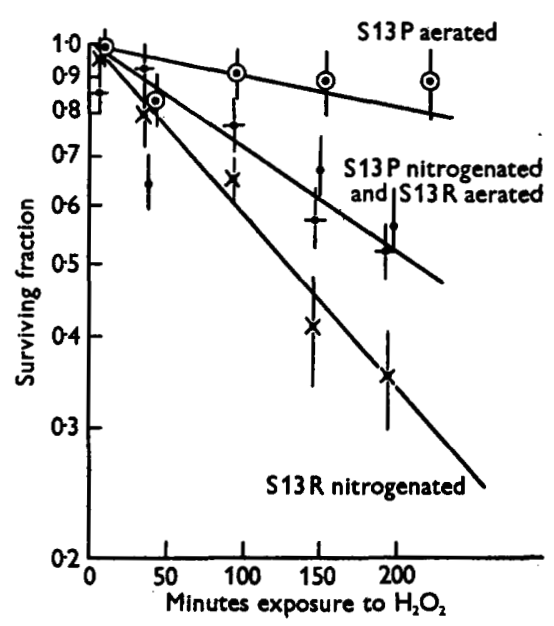

Fig. 2. Effect on phage S13P (poor medium) and S13R (rich medium) of $\mathrm{H}_{2} \mathrm{O}_{2}$; under air or nitrogen bubbling.

Fig. 1. Effect on coli phage $\mathrm{T3}$ of radiationproduced and commercial $\mathrm{H}_{2} \mathrm{O}_{2}$.

Source of hydrogen peroxide. With hydrogen peroxide formed by irradiation in clean aerated water or phosphate buffer, the effect on phage was almost always greater than that of an equal concentration of a commercial product, even of analytical reagent quality several times distilled. An example is illustrated by Fig. 1. The stabilizer contained in commercial hydrogen peroxide apparently inhibited the action of $\mathrm{H}_{2} \mathrm{O}_{2}$ on the phage. Repeated distillation did not entirely remove this stabilizer.

Method of phage stock preparation. Phage S13 when propagated on Evans peptone (phage $\mathrm{S} 13 \mathrm{P}$ ) was found to be less sensitive to $\mathrm{H}_{2} \mathrm{O}_{2}$ than when grown on nutrient agar (phage S 13R) (Fig. 2).

State of aeration of suspension. Hydogen peroxide acted more effectively on phage suspensions from which oxygen was removed by bubbling nitrogen through the suspensions (Fig. 2). 


\section{Effects of other chemical agents}

Ascorbic acid. Ascorbic acid was an inactivating agent for phage, in concentrations somewhat lower than those of $\mathrm{H}_{2} \mathrm{O}_{2}$ which produced the same effect. Survival curves were of exponential form. It was more active in deaerated suspensions, and more active against phage $S 13 R$ than against phage S13P. Dehydroascorbic acid was inactive at comparable concentrations.

Chlorate and iodate. $\mathrm{KClO}_{3}$ and $\mathrm{KIO}_{3}$ were completely inactive against phage in solutions up to $10^{-3} \mathrm{M}$, i.e. in molar concentrations which were considerably higher than those of $\mathrm{H}_{2} \mathrm{O}_{2}$ or ascorbic acid which inactivated the phage.

Urea. Urea has been reported as an agent for breaking hydrogen bonds (Steinhardt, 1938; Alexander \& Stacey, 1952), and for 'splitting' DNA molecules into two (Stacey \& Alexander, 1953). Its action on phage was therefore thought to be of interest. In low concentration urea was inactive; at $4 \mathrm{M}$ (the concentration used by Stacey \& Alexander) it inactivated phage S13, the shape of the survival curve being of non-exponential form (see Fig. 4).

\section{Part inactivation}

It was found that, after $\mathrm{X}$-irradiation of dilute suspensions of shigella phage S 13 or coli phage T3, many of the survivors had suffered a change, although they were still able to adsorb to bacteria and form plaques when plated out immediately. The change manifested itself as a greatly increased susceptibility to the action of hydrogen peroxide or ascorbic acid, but not to heat and urea (Figs. 3, 4). Phage which has been changed in this way by ionizing radiation will be referred to as 'part-inactivated'. The phenomenon did not occur with the survivors of ultraviolet irradiation, nor with phage exposed in concentrated suspension to ionizing radiation, when the inactivating effect of the radiation was of the 'direct' type. It was therefore concluded that part inactivation was due to active radicals formed by the action of radiation on water.

\section{Phage stocks propagated on rich and poor media}

Parallel phage stocks grown on $0.5 \%(\mathrm{w} / \mathrm{v})$ Evans peptone agar (S 13P) and on nutrient agar (S13R) responded differently to some inactivating agents and identically to others. Ultraviolet inactivation and the direct action of $X$-rays were identical for phages S13P and S13R (Figs. 5, 6). On the other hand, the indirect effect of $\mathrm{X}$-rays, i.e. inactivation by active radicals, was much greater with phage $\mathrm{S} 13 \mathrm{R}$ than with $\mathrm{S} 13 \mathrm{P}$ (Fig. 7). The latter effect was also found for stocks of coli phage $\mathrm{T} 3$ propagated on the different media. As mentioned above, S13R was more susceptible than S13P to the action of $\mathrm{H}_{2} \mathrm{O}_{2}$ and ascorbic acid (Fig. 2).

\section{The influence of dissolved gases on radiation effects}

Immediate effect of radiation. It has been shown above that radiation may affect suspended bacteriophage through the $\mathrm{H}_{2} \mathrm{O}_{2}$ formed in aerated aqueous suspensions, and by bringing about part-inactivation, the effects of which are 


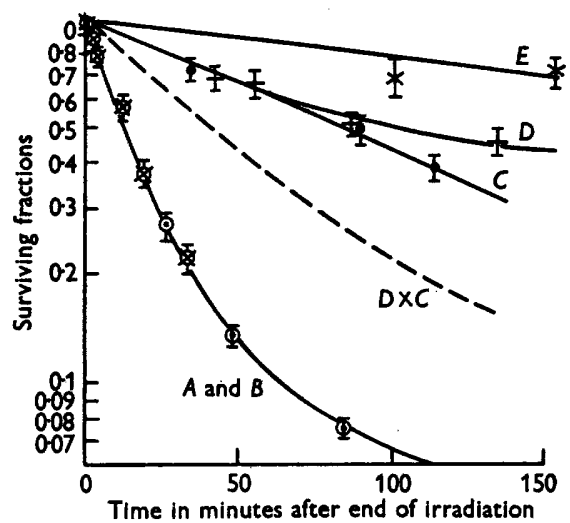

Fig. 3. Effect of irradiated buffer on irradiated and non-irradiated suspensions of shigella phage $\mathrm{S} 13$. $A$, irradiated suspension; $\odot B$, irradiated phage diluted in irradiated buffer; $C$, non-irradiated phage diluted in irradiated buffer $;+D$, irradiated phage diluted in non-irradiated buffer; $\times \boldsymbol{E}$, control.



Fig. 4. Effect of urea on irradiated and non-irradiated suspensions of phage S13.

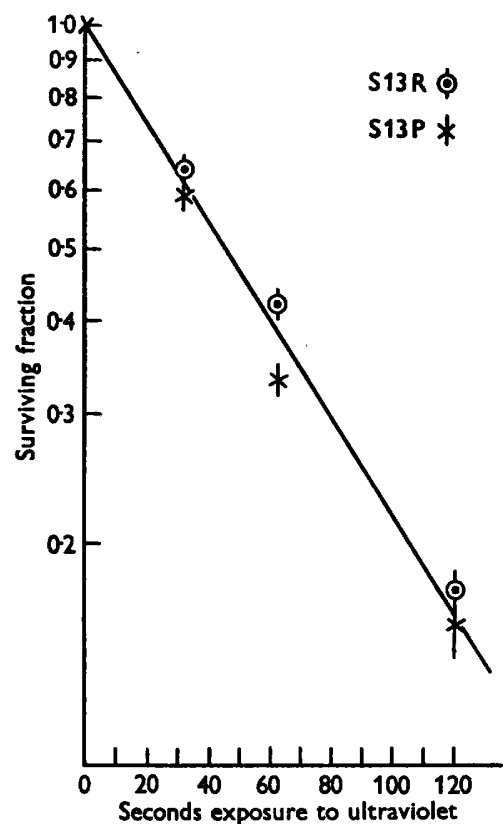

Fig. 5. Effect of ultraviolet radiation on phages S13P and S13R.

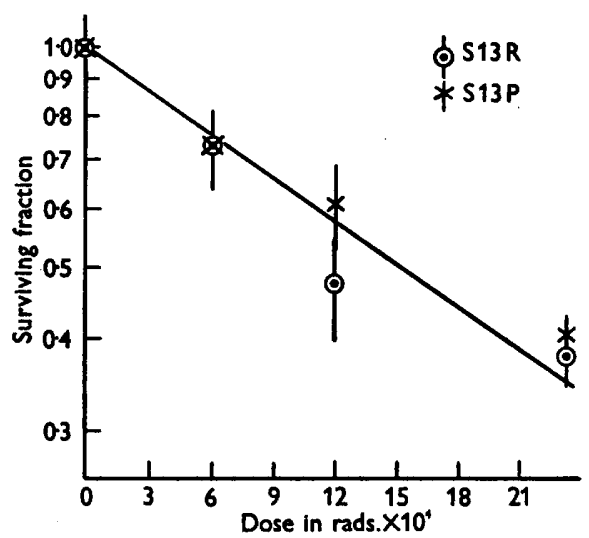

Fig. 6. Direct effect of $X$-rays on phages S13P and S13R. 
persistent and may be followed after the end of the irradiation. Part-inactivation, and the formation of $\mathrm{H}_{2} \mathrm{O}_{2}$, occur as a result of reactions with and between the short-lived products of irradiation, generally agreed to be free radicals, which are present only while the irradiation is proceeding. These radicals may themselves inactivate the phage during irradiation, and this inactivation will be called the immediate effect of radiation. It has been found possible to investigate this alone by delivering radiation in a time which is short compared with that needed for the action of the formed $\mathrm{H}_{2} \mathrm{O}_{2}$, and by sampling the phage

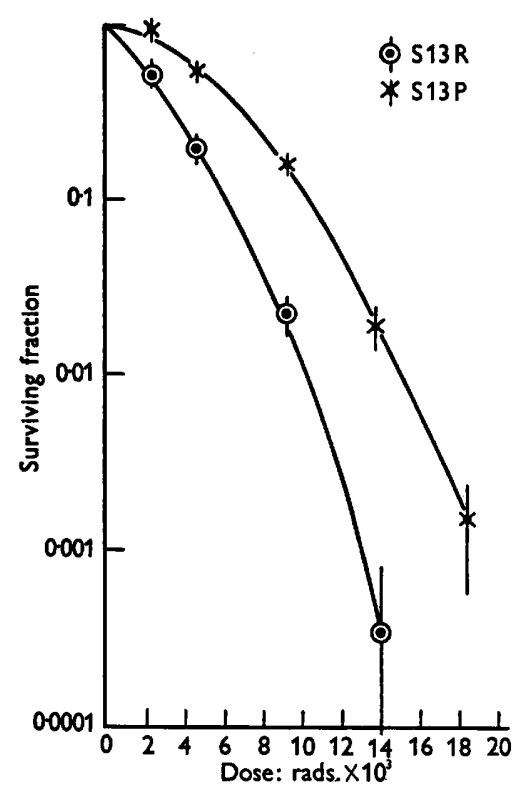

Fig. 7. Indirect effect of X-rays on phages S13P and S13R.

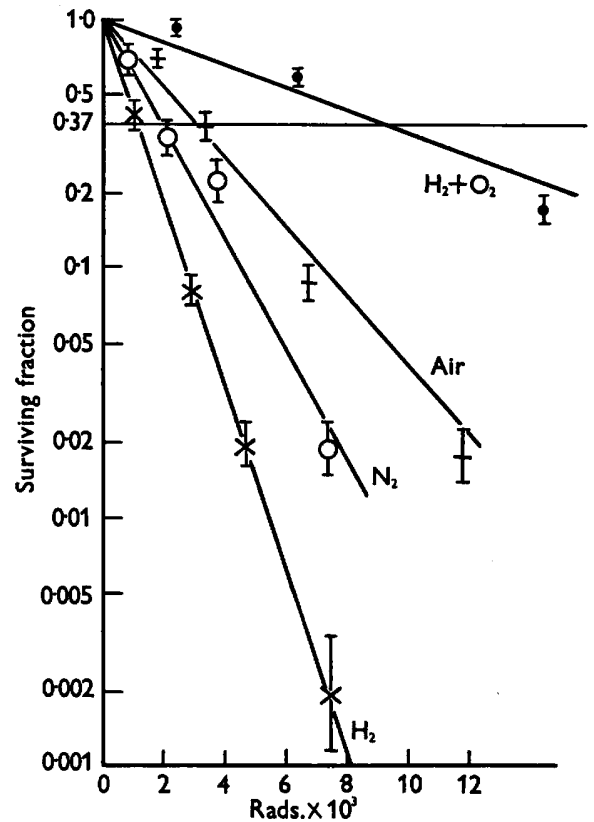

Fig. 8. Influence of various gas treatments on indirect inactivation by $\mathbf{X}$-rays.

very soon after the end of an irradiation. The mechanisms of radical action on phage have to some extent been elucidated by studying the effectiveness of radiation delivered at a high dose rate under the influence of various gas treatments. It was found that the immediate effects of radiation were greatest where the gas treatment was such as to make the maximum number of reducing radicals available to the phage. Fig. 8 illustrates an experiment in which the survival of phage $\mathbf{S} 13$ was followed when it was irradiated in the presence of hydrogen, nitrogen, air, and a mixture of hydrogen and oxygen. The probable nature of the reducing and oxidizing radicals formed under these treatments has been discussed elsewhere (Ebert \& Alper, 1954). The radiation was about ten times as effective under hydrogen bubbling, where reducing reactions were favoured, as under hydrogen +oxygen bubbling, where reducing radicals were rapidly removed and made unavailable to the phage.

Part-inactivation. In aqueous suspensions from which oxygen has been 
removed, $\mathrm{H}_{2} \mathrm{O}_{2}$ is formed by radiation in negligible amounts (Bonet-Maury, 1952). The occurrence of part-inactivation in an air-free suspension would therefore not become manifest unless the survivors of the irradiation were exposed to $\mathrm{H}_{2} \mathrm{O}_{2}$ or ascorbic acid. Experiments were done to investigate the relative extent of part-inactivation occurring in aerated and air-free suspensions. Two phage suspensions and two equal volumes of buffer were irradiated, one of each under aeration, and the other air free. At the end of the irradiation, the air-free buffer was added to the irradiated aerated phage suspension, and the aerated buffer to the air-free phage suspension. Thus the survivors in both suspensions were exposed, after irradiation, to equal con-

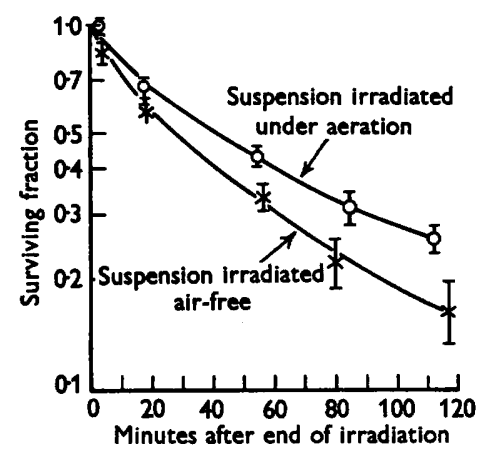

Fig. 9. Post irradiation effect of $\mathrm{H}_{2} \mathrm{O}_{2}$ on suspensions of phage $\mathrm{S} 13$ irradiated with and without aeration.

centrations of $\mathrm{H}_{2} \mathrm{O}_{2}$. As can be seen from Fig. 9, the inactivation of the suspension which had been irradiated in the absence of oxygen was somewhat more rapid. Thus part-inactivation, as well as immediate inactivation, appears to arise from reaction with reducing radicals.

\section{Effects of hydrogen peroxide under irradiation}

When phage was introduced into previously irradiated buffer and then exposed to further ionizing radiation, the total effect was greater than the sum of the effects of radiation and peroxide acting independently. In a typical experiment phosphate buffer ( $\mathrm{pH} 7.0 ; 0.001 \mathrm{M})$ was irradiated with 11,000 roentgens of $\gamma$-rays, a dose which formed about $1 \mu \mathrm{g} . \mathrm{H}_{2} \mathrm{O}_{2} / \mathrm{ml}$. buffer. Phage was introduced into two ampoules (1) and (2) containing the irradiated buffer; ampoule (1) was then subjected to further $\gamma$ irradiation. In addition, phage was irradiated by the same source of $\gamma$-rays without previous irradiation of the buffer (ampoule (3)). The three survival curves are presented together in Fig. 10. The effect of irradiating the phage in the previously irradiated buffer was considerably greater than the sum of the separate effects of irradiated buffer and irradiation, particularly in the earlier stages of irradiation, when no appreciable amount of $\mathrm{H}_{2} \mathrm{O}_{2}$ had yet been formed in ampoule (3). By contrast, the effect of ultraviolet light on phage in the presence of $\mathrm{H}_{2} \mathrm{O}_{2}$ was no greater than the sum of the separate effects of the $\mathrm{H}_{2} \mathrm{O}_{2}$ and the ultraviolet radiation. 


\section{DISCUSSION}

The majority of biological radiation effects appear to be oxidative in nature (Gray, 1953) as judged mainly by the fact that the presence of oxygen enhances the effectiveness of the radiation. The radiation experiments with phage show that with this system reducing radicals are the inactivating agents, although the presence of oxygen during radiation might give rise to enhanced effects through the formation of hydrogen peroxide, if part-inactivation has

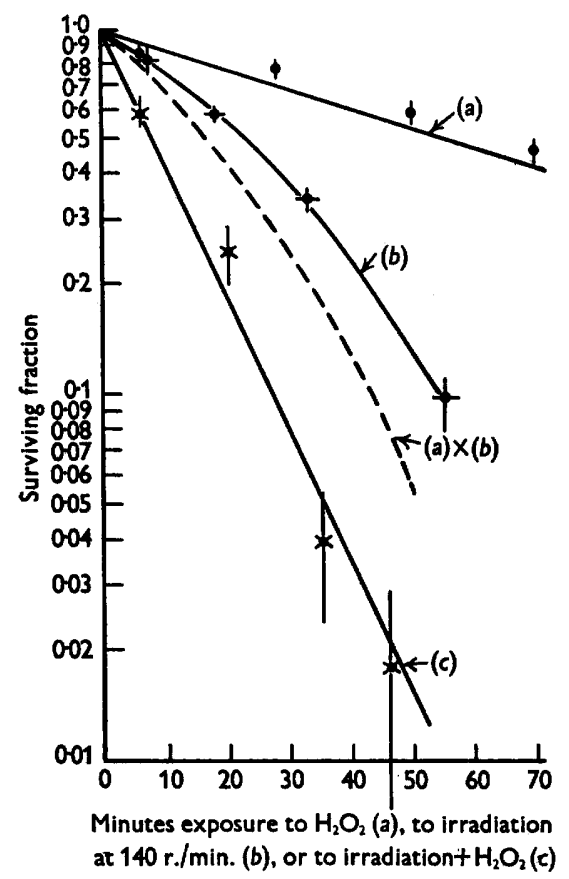

Fig. 10. Effect of $\mathrm{H}_{2} \mathrm{O}_{2}$ on phage $\mathrm{S} 13$ undergoing further irradiation.

taken place and if there is a time lapse between the radiation period and the time of sampling. That radiation reduction processes in other biological systems might be disguised as oxidations, because of oxygen enhancement, is suggested by work of Daniels, Scholes \& Weiss (1953). These authors found that the viscosity of thymus deoxyribosenucleic acid was immediately affected by radiation to a greater extent in the absence of oxygen than in its presence; but the after-effects were considerably more when oxygen was present during irradiation.

The conclusion that free phage is very sensitive to reducing agents is supported by the inactivating effect of ascorbic acid and by the lack of effect of oxidizing agents in concentrations of much higher molarity. It is usually assumed that $\mathrm{H}_{2} \mathrm{O}_{2}$ acts on biological systems by virtue of its oxidizing properties, but it can also act as a reductant, as with strong oxidizing agents such as $\mathrm{KMnO}_{4}$. That $\mathrm{H}_{2} \mathrm{O}_{2}$ acts as a reductant on bacteriophage is inferred from 
the experimental work with other reducing agents, and also from the fact that the effects of $\mathrm{H}_{2} \mathrm{O}_{2}$ on phage suspensions are enhanced when oxygen is removed by the passage of nitrogen through the suspension. From experiments in which $\mathrm{H}_{2} \mathrm{O}_{2}$ which had not been freed from stabilizer was used, it is inferred that before $\mathrm{H}_{2} \mathrm{O}_{2}$ is effective in inactivating phage it must decompose. $\mathrm{H}_{2} \mathrm{O}_{2}$ decomposition is brought about by radiation, and under $\gamma$-irradiation the effect of the $\mathrm{H}_{2} \mathrm{O}_{2}$ was much greater than without irradiation, allowing for inactivation due to the $\gamma$-irradiation. With decomposition of hydrogen peroxide by ultraviolet radiation, on the other hand, no enhanced effect on the phage was observed.

Under ultraviolet irradiation, the reaction: $\mathrm{H}_{2} \mathrm{O}_{2} \rightarrow \mathrm{OH}+\mathrm{OH}$, takes place (Urey, Dawsey \& Rice, 1929). The hydroxyl radicals may in their turn react with $\mathrm{H}_{2} \mathrm{O}_{2}$ molecules, producing free radicals of other types. The steps in $\mathrm{H}_{2} \mathrm{O}_{2}$ decomposition under ionizing radiation are not known, but from the present work it may be inferred that the proportion of reducing radicals made available during ionizing irradiation is very much higher than with ultraviolet irradiation. In experiments previously reported (Alper, 1952b) it was shown that when an aerated phage suspension was irradiated by $\gamma$-rays at a low dose rate in the presence of catalase, the shape and slope of the survival curve indicated that the catalase protected the phage by decomposing the formed $\mathrm{H}_{2} \mathrm{O}_{2}$. This protection could be given only if the catalase decomposed the $\mathrm{H}_{2} \mathrm{O}_{2}$ via harmless radicals, as in fact is likely in ordinary biological decomposition of $\mathrm{H}_{2} \mathrm{O}_{2}$, whereas without catalase present the $\mathrm{H}_{2} \mathrm{O}_{2}$ could attack the phage by decomposition through other reactions. The decomposition of $\mathrm{H}_{2} \mathrm{O}_{2}$ by catalase was considered by Weiss (1935) to proceed via $\mathrm{OH}$ radicals.

The reduction of phage particles was observed in those conditions in which reactions took place with inactivating agents arising from, or dissolved in, the suspending medium; this suggests the possibility of reactions at the surface of the particles. The phenomena associated with reducing processes (protective effect of oxygen, enhanced sensitivity to ascorbic acid) do not arise under the direct action of $\mathrm{X}$-rays, nor with inactivation by ultraviolet radiation, with which there is no 'indirect effect' since, at the wave lengths used, the energy is not absorbed in the suspending medium. Watson (1952) suggested that the mechanism of inactivation by indirect action of $\mathbf{X}$-rays might be different from that of direct action. He obtained some evidence that phage inactivated by indirect action failed to adsorb, whereas with ultraviolet or direct $\mathbf{X}$-ray inactivation (Watson, 1951) adsorption is unimpaired. It seems likely that the inactivation of phage by reducing agents arises from an interference with one of the steps in adsorption to the bacterial host. (Puck, Garen \& Cline, 1951; Garen \& Puck, 1951; Tolmach \& Puck, 1952). Experiments are planned which, it is hoped, will yield further information on this point.

The part-inactivation phenomenon, which itself occurs under irradiation as a result of reaction with reducing radicals, suggests that the reduction of a phage particle may take place in two steps, the first of which does not impair its ability to adsorb to bacteria but does increase the probability of a second reaction with a reducing agent. A phage population which has survived the 
immediate effects of a small dose of radiation is composed of part-inactivated particles and of those which have escaped any action of the free radicals. Initially the action of a post-irradiation inactivating agent proceeds rapidly, until only those particles are left which are inactivated at the same rate as those in non-irradiated suspensions. Post-irradiation survival curves can in fact be resolved into two exponential curves, the less steep one being parallel to the survival curve of non-irradiated phage exposed to the same agent (Fig. 11). It can be inferred that inactivation by reducing agents is a first order reaction, the probability of its occurrence at any collision being greatly increased with part-inactivated phage.

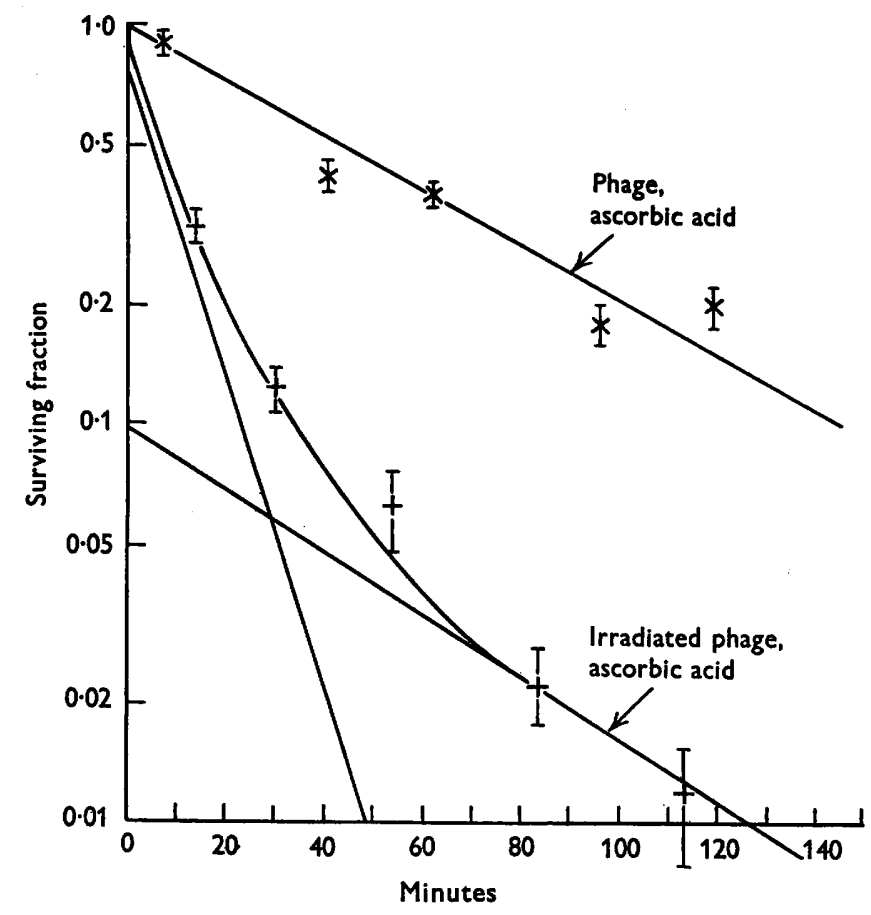

Fig. 11. Effect of ascorbic acid on irradiated and non-irradiated suspensions of phage $\mathbf{S 1 3}$.

Inactivation by agents such as ultraviolet radiation and the direct action of X-rays proceeds at the same rate for phage S13P (propagated on poor medium) as for phage $\mathbf{S} 13 \mathrm{R}$ (rich medium). Inactivation by reducing agents, on the other hand, proceeds faster with S 13R. Cohen (1947) described different inactivation rates of stocks of coli phage $\mathrm{T} 2$ grown on rich or poor media, and concluded that the difference arose from the fact that phage particles grown on poor media were coated with a layer of deoxyribosenucleic acid, probably of bacterial origin. Experiments have not yet been done to see whether the differences between S13P and S13R disappear after digestion with deoxyribonuclease. It is noteworthy, however, that by contrast with coli phage T2 grown by Cohen on poor media, S13P stocks have remained 


\section{Phage inactivation by reducing agents}

stable over many months. Moreover, whenever there were differences in the responses of phages $S 13 P$ and $S 13 R$ to inactivating agents, the former was more resistant, in contrast to Cohen's findings with coli phage T2. If these differences between S13P and S13R are in fact due to the presence of a deoxyribonucleic acid layer on the surface of S13P, it would follow that this layer protects against reduction and is itself sensitive to reducing agents.

I am greatly indebted to Dr M. Ebert for active collaboration in some of the experiments here described and for his unfailing assistance during much of the investigation. It is a pleasure to acknowledge the stimulating effects of discussions with Dr L. H. Gray and Dr William Hayes. Most of the work was done while I was in receipt of a personal research grant from the Medical Research Council.

\section{REFERENCES}

Alexander, P. \& Stacey, K. A. (1952). The colloidal behaviour of dyes. Proc. Roy. Soc. A, 212, 274.

Alper, T. (1952 a). A new after-effect of X-rays on dilute aqueous suspensions of bacteriophage. Nature, Lond. 169, 964.

ALPER, T. (1952 b). Indirect inactivation of bacteriophage during and after exposure to ionizing radiation. Radiation Chemistry; General Discussions, Faraday Soc. no. 12, 234 .

AlPER, T. (1954). Inactivation of bacteriophage after irradiation. 1952 Symposium, Faraday Soc., on Chemistry of Biological After-effects of Ultraviolet and Ionizing Radiations. Brit. J. Radiol. 27, 50.

Bonet-MaURy, P. (1952). Chemical phenomena in irradiated pure water. Radiation Chemistry; General Discussions, Faraday Soc. no. 12, 72.

CoHEN, S. S. (1947). Streptomycin and deoxyribonuclease in the study of variations in the properties of a bacterial virus. J. biol. Chem. 168, 511.

Daniels, M., Scholes, G. \& Weiss, J. (1953). 'After-effect' in aqueous solutions of deoxyribonucleic acid irradiated with X-rays. Nature, Lond. 171, 1153.

EBERT, M. \& ALPER, T. (1954). Influence of dissolved gases on hydrogen peroxide formation and bacteriophage inactivation by radiation. Nature, Lond. 173, 987.

EisenderG, G. M. (1943). Colorimetric determination of hydrogen peroxide. Industr. Engng Chem. (Anal.), 15, 327.

Garen, A. \& Puck, T. (1951). The first two steps of the invasion of host cells by bacterial viruses II. J. exp. Med. 94, 177.

Gray, L. H. (1953). The initiation and development of cellular damage by ionizing radiations. Brit. J. Radiol. 26, 609.

Lea, D. E. \& Salaman, H. M. (1946). Experiments on the inactivation of bacteriophage by radiations, and their bearing on the nature of bacteriophage. Proc. Roy. Soc. B, 133, 434.

Puck, 'T., Garen, A. \& Cline, J. (1951). The mechanism of virus attachment to host cells. I. The role of ions in the primary reaction. J. exp. Med. 93, 65.

SAVAGE, D. J. (1951). The determination of hydrogen peroxide in radiation experiments on aqueous solutions. Analyst, 76, 224.

Stacey, K. A. \& Alexander, P. (1953). Evidence for the twin spiral structure proposed for DNA. Abstract, Faraday Soc. Informal Discussion on Structure and Properties of Nucleic Acid and Nucleoproteins.

Steinhardt, J. (1938). Properties of haemoglobin and pepsin in solutions of urea and other amides. J. biol. Chem. 123, 543.

Tolmach, L. J. \& Puck, T. (1952). The mechanism of virus attachment to host cells. III. J. Amer. chem. Soc. 74, 5551. 
Urey, H. C., Dawsey, L. H. \& Rice, F. O. (1929). The absorption spectrum and decomposition of hydrogen peroxide by light. J. Amer. chem. Soc. 51, 1371.

WATSON, J. (1951). The properties of X-ray inactivated bacteriophage. I. Inactivation by direct effect. J. Bact. 60, 697 .

WATson, J. (1952). The properties of X-ray inactivated bacteriophage. II. Indirect effects of radiation. $J$. Bact. $63,473$.

WeIss, J. (1935). The catalytic decomposition of hydrogen peroxide on different metals. Trans. Faraday Soc. 31, 1547.

(Received 17 May 1954) 